\title{
Wheat seeds can delay orthodontic tooth movement by blocking osteoclastogenesis in rats
}

This article was published in the following Dove Press journal:

Clinical, Cosmetic and Investigational Dentistry

\section{Sri Suparwitri \\ Niswati Fathmah Rosyida \\ Ananto Ali Alhasyimi}

Department of Orthodontics, Faculty of Dentistry, Universitas Gadjah Mada,

Yogyakarta, Indonesia
Correspondence: Ananto Ali Alhasyimi Department of Orthodontics, Faculty of Dentistry, Universitas Gadjah Mada,

Yogyakarta 5528I, Indonesia

Tel +62 82136708250

Email anantoali@ugm.ac.id
Background: The hormone estrogen can prevent osteoclast differentiation and suppress osteoclastic activity, which can prevent tooth relapse movement. Wheat seeds are a natural material containing phytoestrogens with similar characteristics to estrogen.

Objective: This study intends to determine the influence of wheat seeds in reducing osteoclastogenesis following orthodontic tooth movement (OTM).

Materials and methods: Forty male Sprague Dawley rats were used in this experimental study. The rats were divided into control and treatment groups $(n=20)$. In both groups, the rat maxillary incisors were moved distally using a $0.012 \mathrm{U}$ stainless steel orthodontic appliance with a $2 \mathrm{~mm}$ diameter coil and $5 \mathrm{~mm}$ wire length. The treatment group received wheat seeds at a dose of 0.6 gram/day. The animals were sacrificed at days $0,1,7$, and 14 after using the orthodontic appliance (4 subgroups according to observations of days; $n=5$ ). RANKL and OPG expressions were determined from the gingival crevicular fluid (GCF) samples using ELISA analysis. Statistical analysis of the data collected was calculated with the independent sample $t$-test $(P<0.05)$.

Results: All the experimental procedures were well-tolerated and did not change the animals' weight. On days 7 and 14, the OTM rate of the treatment group was significantly lower than the control group $(P<0.05)$. ELISA test results showed that the OPG level was significantly higher on days 1,7 , and 14 , while the RANKL level was considerably lower on day 14 in the wheat seeds group compared with the control group $(P<0.05)$.

Conclusion: Wheat seeds can promote osteoclastogenesis inhibition and delay OTM.

Keywords: wheat seeds, estrogen, relapse, orthodontic

\section{Introduction}

Long-term stability post-orthodontic treatment is controversial among orthodontists. ${ }^{1}$ Uncompleted alveolar bone remodeling is one of the etiological factors of orthodontic relapse. ${ }^{2}$ Orthodontic tooth movement (OTM) is characterized by periodontal tissue bone remodeling processes. Bone remodeling is a process where mature bone tissue is displaced, and new bone tissue is created, which is supported by osteoclasts and osteoblasts, through a process called osteoclastogenesis. This process involves an interaction between the receptor activator of nuclear factor- $\kappa \mathrm{B}$ ligand (RANKL), receptor activator of nuclear factor- $\mathrm{B}$ (RANK), and osteoprotegerin (OPG). The communication within RANK exposed to osteoclast precursors and RANKL exposed to osteoblasts is fundamental for osteoclast differentiation and activation. On the other hand, osteoprotegerin (OPG) expressed by osteoblast will inhibit RANK and RANKL binding to prevent osteoclast differentiation. ${ }^{3}$ During a relapse, RANKL levels will elevate, while OPG levels will decrease. ${ }^{1}$ 
Several methods to influence tooth movement by controlling bone remodeling have been developed to prevent relapse, including the use of hydrogel carbonate apatiteincorporated advanced platelet-rich fibrin. ${ }^{4}$ However, the use of natural materials is preferred due to its minimal side effect. ${ }^{5}$ Wheat seeds are a natural material that can be found easily everywhere. Wheat seeds contain $379,380 \mu \mathrm{g} / 100 \mathrm{~g}$ of phytoestrogens, which acts like non-steroidal natural products that mimic the estrogen hormone by binding to estrogen receptors. ${ }^{6}$ Estrogen is known to stimulate OPG secretion from osteoblasts and inhibits RANKL production. This phenomenon can affect the anti-resorption properties of estrogen. Frequently, estrogen production is comparable to OPG secretion, and a reduced estrogen production has prevented OPG production as well. ${ }^{7}$ Thus, this present study aims to investigate the potential of wheat seeds for slowing OTM and blocking osteoclastogenesis in rats whose teeth were moved orthodontically. The null hypothesis to be tested was that wheat seeds extract administration would delaye the orthodontic tooth movement by blocking osteoclastogenesis in rats.

\section{Materials and methods}

\section{Animal study}

Ethical consent was received from the Research Ethics Committee of the Faculty of Dentistry, University Gadjah Mada, Yogyakarta, Indonesia, following the ARRIVE
(Animal Research: Reporting of In Vivo Experiments) guidelines, with number 001461/KKEP/FKG-UGM/EC/ 2018. Forty 10-week-old male Sprague Dawley rats were divided randomly into a control and treatment group. Then the two groups were divided into four subgroups according to the observation days $0,1,7$, and 14 with 5 rats in each group. A three-spin loop spring was made with 0.012 stainless steel wire, $2 \mathrm{~mm}$ diameter coil, $5 \mathrm{~mm}$ wire arm length and was placed in the right and left maxillary incisors (Figure 1). Rats were anesthetized with ketamine and xylazine (ketamine dose $35 \mathrm{mg} / \mathrm{kg}$ BB and xylazine $5 \mathrm{mg} / \mathrm{kg}$ BB) intramuscularly when the orthodontic appliance was placed in their mouth. The 3-spin loop spring was cemented to the maxillary incisor to deliver 35 grams $(35 \mathrm{cN})$ of orthodontic mechanical induction. In the treatment group, a wheat seeds solution $(0,6 \mathrm{~g}$ in $3 \mathrm{ml}$ of aquadest) was administrated since the day the orthodontic device was applied until the 1st, 4th, 7th, and 14th days. The solution was given orally once a day using a nasogastric tube.

\section{Orthodontic tooth movement measurement}

The measurement of tooth movement was determined using a sliding caliper with a precision level of $0.01 \mathrm{~mm}$ on days $0,1,7$, and 14 after insertion of the orthodontic device. Measurements were taken by one observer blinded to the experimental groups. The examiner showed a good level agreement in his analysis $(\kappa=0.87)$, indicating

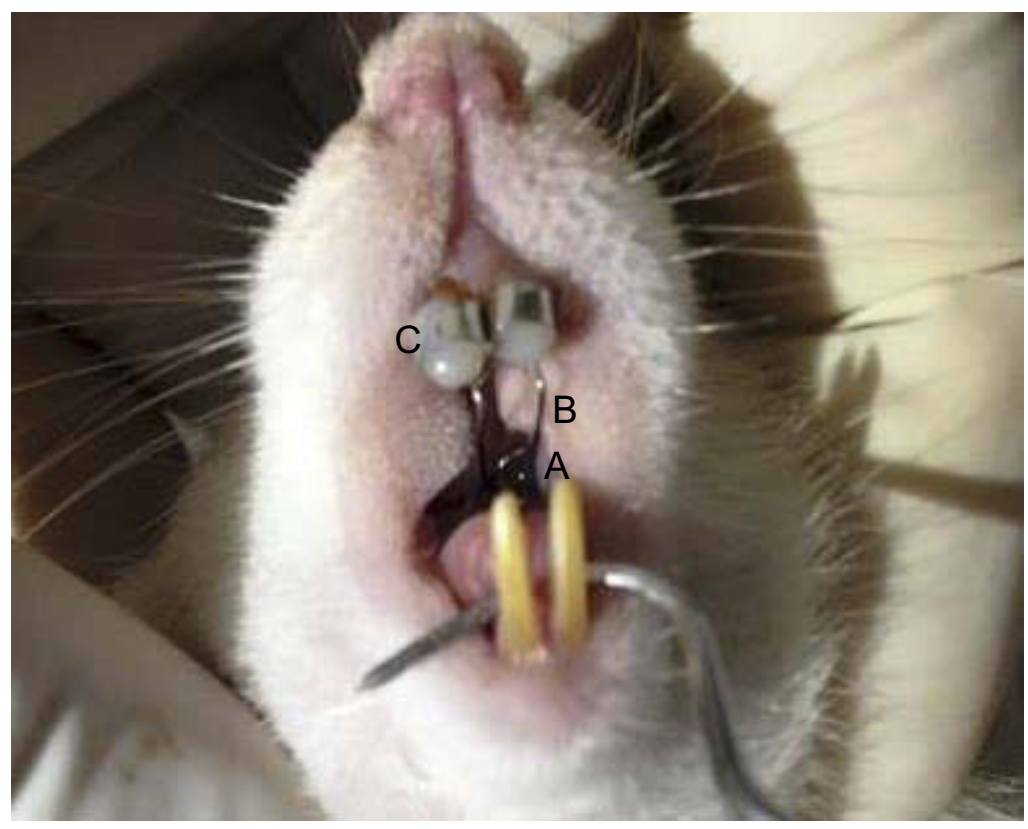

Figure I Design of the orthodontic device: (A) coil, (B) arm, and (C) matrix band. 
satisfactory intra-examiner reliability. The measurements were repeated three times and then averaged.

\section{Isolation of gingival crevicular fluid}

The collection of GCF was taken using paper point size 15 in each group on days $1,4,7$, and 14. To eliminate the residual saliva and other oral fluid, during GCF sample collection, each crevicular sulcus of the animal was lightly drained by air jets and isolated with cotton rolls. A periodontal paper was introduced into the mesial sulcus of the incisor teeth periodontal gingiva (tension side) for $45 \mathrm{sec}$ onds (Figure 2). Afterward, the paper point was put in a $1.5 \mathrm{ml}$ Eppendorf tube containing $350 \mu \mathrm{l}$ of physiological saline solution. The Eppendorf tube was centrifuged for 5 mins at a speed of $2000 \mathrm{rpm}$ to elute the GCF component completely. Paper points were taken, and the supernatant solution was stored at $-80{ }^{\circ} \mathrm{C}$ until analysis.

\section{Analysis with enzyme-linked immunosorbent assay (ELISA)}

Detection of RANKL and OPG levels were measured using the ELISA (enzyme-linked immunosorbent assay) test. The analysis was applied using a quantitative sandwich Rat ELISA kit specific for each protein (FineTest Co. Ltd., China). Anti- OPG and sRANKL antibody were pre-coated onto 96-well plates. And the biotin-conjugated antisRANKL antibody was used as detection antibodies. HRP-Streptavidin conjugate was mixed into the plates and unchained conjugates were cleaned away by wash buffer. TMB substrates were used to visualize HRP enzymatic effect. TMB was catalyzed by HRP to produce a blue color product that turned into yellow after adding acidic stop solution. The measurements of optical density used spectrophotometry microplate reader at a wavelength of $450 \mathrm{~nm}$. The concentration of RANKL and OPG in the sample was determined by comparing the optical density of the sample to the standard curve. The total amount of RANKL and OPG expression level were revealed as $\mathrm{pg} / \mathrm{mL}$ ( $\mathrm{pg}=\operatorname{picogram} / \mathrm{mL}=$ milliliter $)$.

\section{Statistical analysis}

To identify possible differences in OTM rate, RANKL and OPG expression between controls and treatment groups, an independent sample $t$-test was used since the

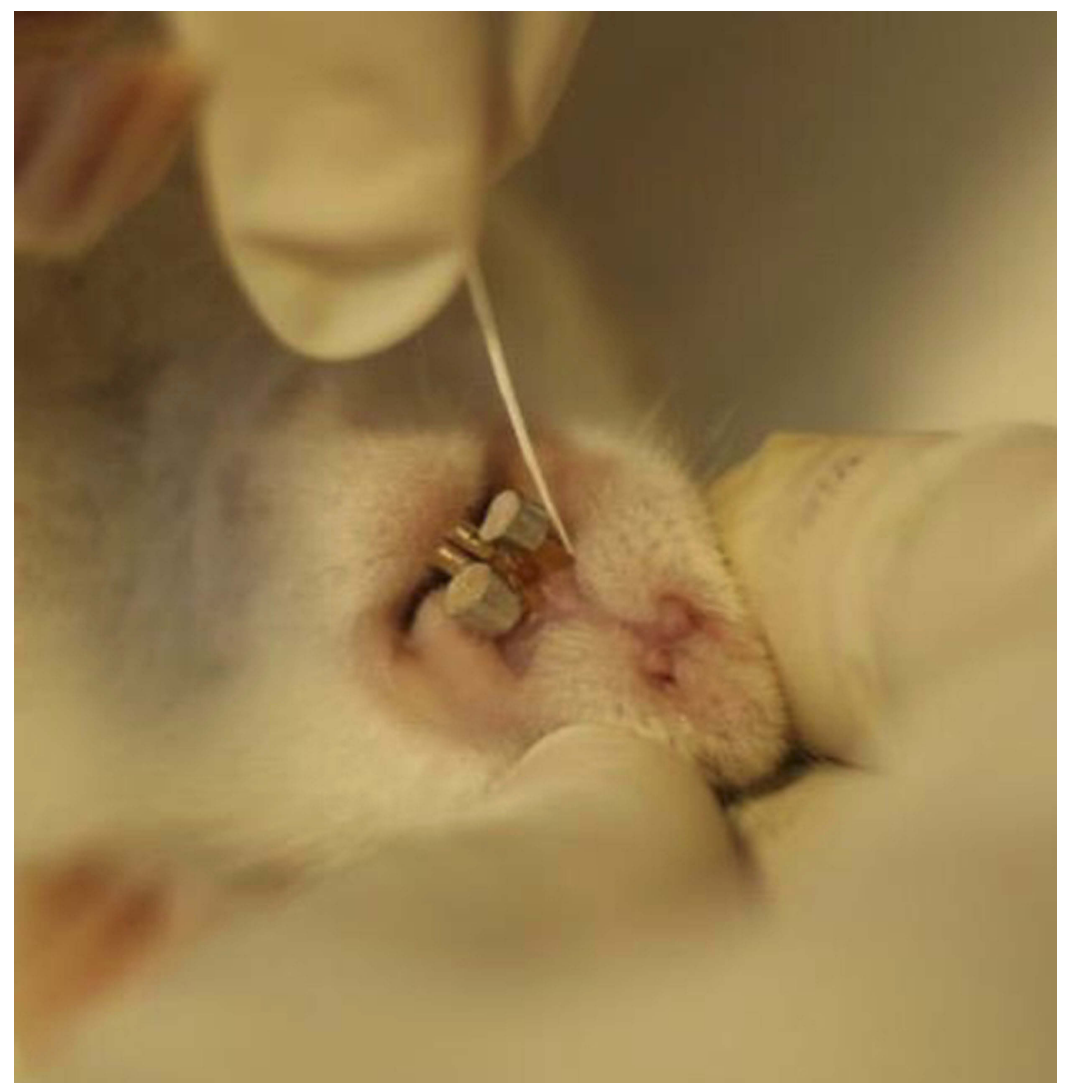

Figure 2 Gingival crevicular fluid isolation. 
distribution of the data was normal and the data was homogen. Statistical significance was considered when $P$ values $<0.05$.

\section{Results}

In general, wheat seeds administration at the selected dosage did not cause any systemic toxicity or changed the body volume of the rats. The correlation between groups demonstrated a significant difference among the control groups and those that received wheat seeds. Regarding the clinical observation, the OTM speed range of the groups receiving wheat seeds was significantly lower than the OTM speed range of the control group on days 7 , and 14 following appliance bonding $(P<0.05)$. Then the ELISA analysis revealed that the OPG level was significantly higher on days 1, 7, and 14 after appliance bonding and the RANKL level was significantly lower on day 14 after appliance bonding in the wheat seeds group compared with the control group $(P<0.05)$ (Table 1, Figures 3-5).

\section{Discussion}

Orthodontic treatment requires a retention period in which the new position is maintained and stabilized. In this

Table I Differences of OPG and RANKL levels $(\mathrm{pg} / \mathrm{ml})$ and OTM rates $(\mathrm{mm})$ at specific measurement timepoints between the 2 groups examined

\begin{tabular}{|l|l|l|l|}
\hline & $\begin{array}{l}\text { Control } \\
\text { Group } \\
\text { (CG) }\end{array}$ & $\begin{array}{l}\text { Wheat seed } \\
\text { Group (WS) }\end{array}$ & $\begin{array}{l}\text { P- } \\
\text { value }^{\mathbf{a}}\end{array}$ \\
\hline OTM rates (mm) & $0.401 \pm 0.107$ & $0.426 \pm 0.158$ & 0.805 \\
0 & $0.693 \pm 0.186$ & $0.712 \pm 0.145$ & 0.143 \\
I & $1.214 \pm 0.364$ & $0.902 \pm 0.416$ & $0.049^{*}$ \\
7 & $1.627 \pm 0.591$ & $1.017 \pm 0.499$ & $0.043^{*}$ \\
\hline 14 & & & \\
\hline OPG levels (pg/ml) & $0.216 \pm 0.158$ & $0.251 \pm 0.111$ & 0.169 \\
0 & $0.178 \pm 0.169$ & $0.213 \pm 0.117$ & $0.046^{*}$ \\
I & $0.186 \pm 0.151$ & $0.251 \pm 0.113$ & $0.018^{*}$ \\
7 & $0.207 \pm 0.195$ & $0.269 \pm 0.109$ & $0.049^{*}$ \\
\hline 14 & & & \\
\hline RANKL levels (pg/ml) & & & \\
0 & $0.237 \pm 0.103$ & $0.259 \pm 0.167$ & 0.364 \\
I & $0.278 \pm 0.143$ & $0.262 \pm 0.152$ & 0.759 \\
7 & $0.314 \pm 0.158$ & $0.248 \pm 0.104$ & 0.198 \\
I4 & $0.271 \pm 0.156$ & $0.206 \pm 0.102$ & $0.048^{*}$ \\
\hline
\end{tabular}

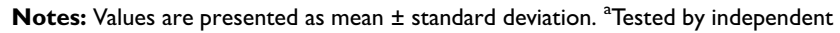
$t$-test of variance. ${ }^{*} P<0.05$, significant differences between groups.

Abbreviations: OTM, orthodontic tooth movement; RANKL, receptor activator of nuclear factor- $\kappa B$ ligand; OPG,osteoprotegerin. retention period, the alveolar bone is remodeled and settle in its new position to avoid a shift toward the initial pretreatment position. The conventional approaches to orthodontic retention include the use of fixed or removable retainers, which are heavily influenced by the level of patient cooperation. Orthodontic treatment can yield reasonably good long-term stability using biological retainers. To explore this topic, a natural material from wheat seed has been introduced. In this study, we assessed the influence of wheat seed on OTM and discovered it has a blocking effect on tooth movement in rats. Natural products containing phytoestrogens like wheat seeds could induce osteoblast activation and stimulate new bone formation. Phytoestrogens are essential estrogenic compounds found in many plants and seeds. This compound has a composition similar to mammalian estrogen. ${ }^{8}$ Estrogen has been identified to have a positive effect on bone by inducing osteoblast differentiation through the upregulation of paired basic amino acid-cleaving enzyme 4 (PACE4) expression. PACE4 is a member of the subtilisin-like proprotein convertase, which is required for the maturation/activation of bone formation factors such as bone morphogenetic proteins and osteocalcin. ${ }^{9}$ The groups exposed to wheat seed exhibited significantly $(P<0.05)$ lower RANKL levels, raised OPG levels, and certainly lower OTM than control groups.

Modifications in orthodontic practice have occurred in the last decade, and improvements in therapeutic technologies have open new avenues, which clinicians may use in daily clinical practice. ${ }^{10}$ Wheat seeds are one type of phytoestrogen plant that is known to have content and characteristics similar to the estrogen hormone. Estrogens help maintain normal bone density, and it has been hypothesized that phytoestrogens may present similar characteristics. ${ }^{11}$ The content and characteristics of phytoestrogens, which are similar to estrogen hormone, have a role in inhibiting the Receptor Activator of Nuclear Factor Kappa $\beta$ (RANK) interactions with their ligands, namely the Receptor Activator of Nuclear Factor Kappa $\beta$ Ligand (RANKL). Estrogen stimulates the production of Osteoprotegerin (OPG), also known as osteoclastogenesis inhibitory factor (OCIF), which inhibits the differentiation and activity of osteoclast cells. An improvement in the OPG level secreted by osteoblast induces a reduction in the receptor activator of nuclear factor- $\kappa \mathrm{B}$ ligand level and can restrain OTM. OPG is an essential receptor of osteoblastic cells, which competes with the receptor activator of the nuclear factor- $\kappa \mathrm{B}$ used by osteoclast, restrains osteoclast differentiation and activity by attaching to the receptor activator of 


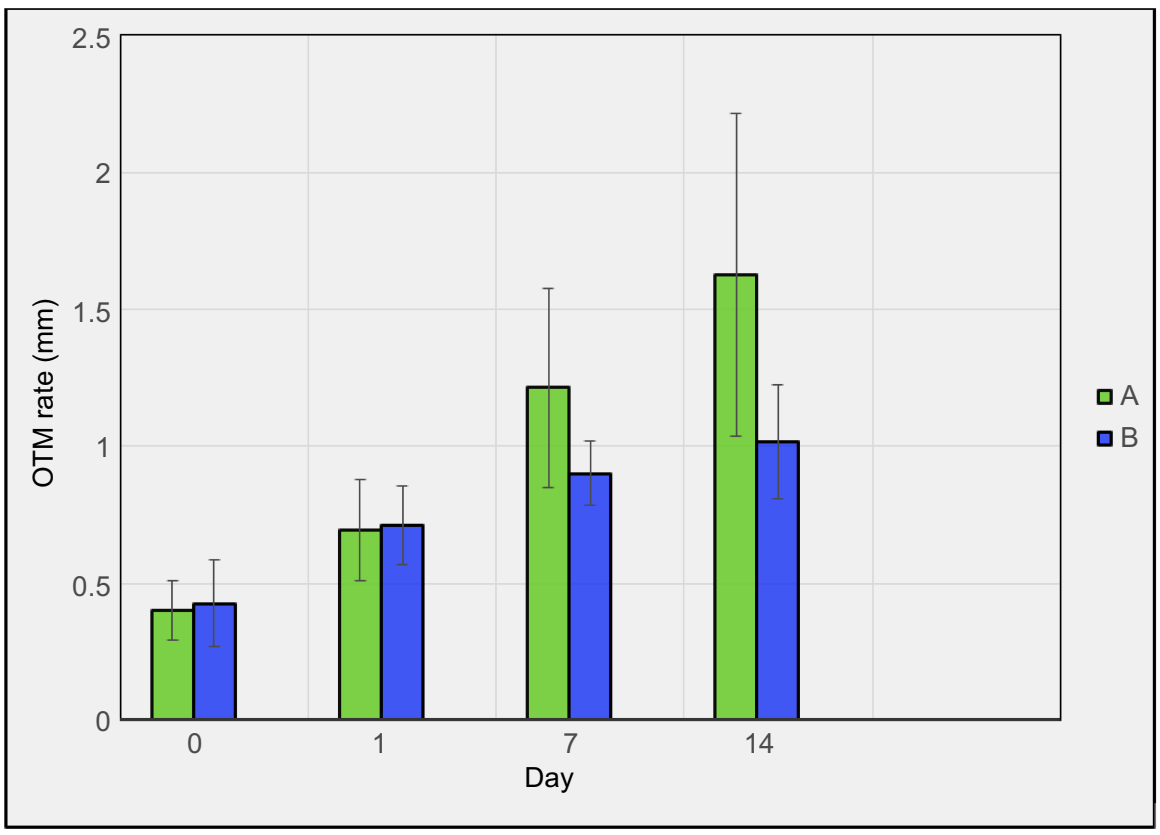

Figure 3 Comparison of orthodontic tooth movement (OTM) rates among 2 groups tested. A indicates control group, B indicates group receiving wheat seed.

nuclear factor- $\mathrm{kB}$ ligand and blocks receptor activator of the nuclear factor- $\mathrm{\kappa B}$ ligand from combining with the receptor activator of nuclear factor- $\mathrm{kB}{ }^{12}$ These previous findings explain our investigation results and describe the connection between wheat seed administration and the reduction of receptor activator of nuclear factor- $\mathrm{kB}$ ligand level.

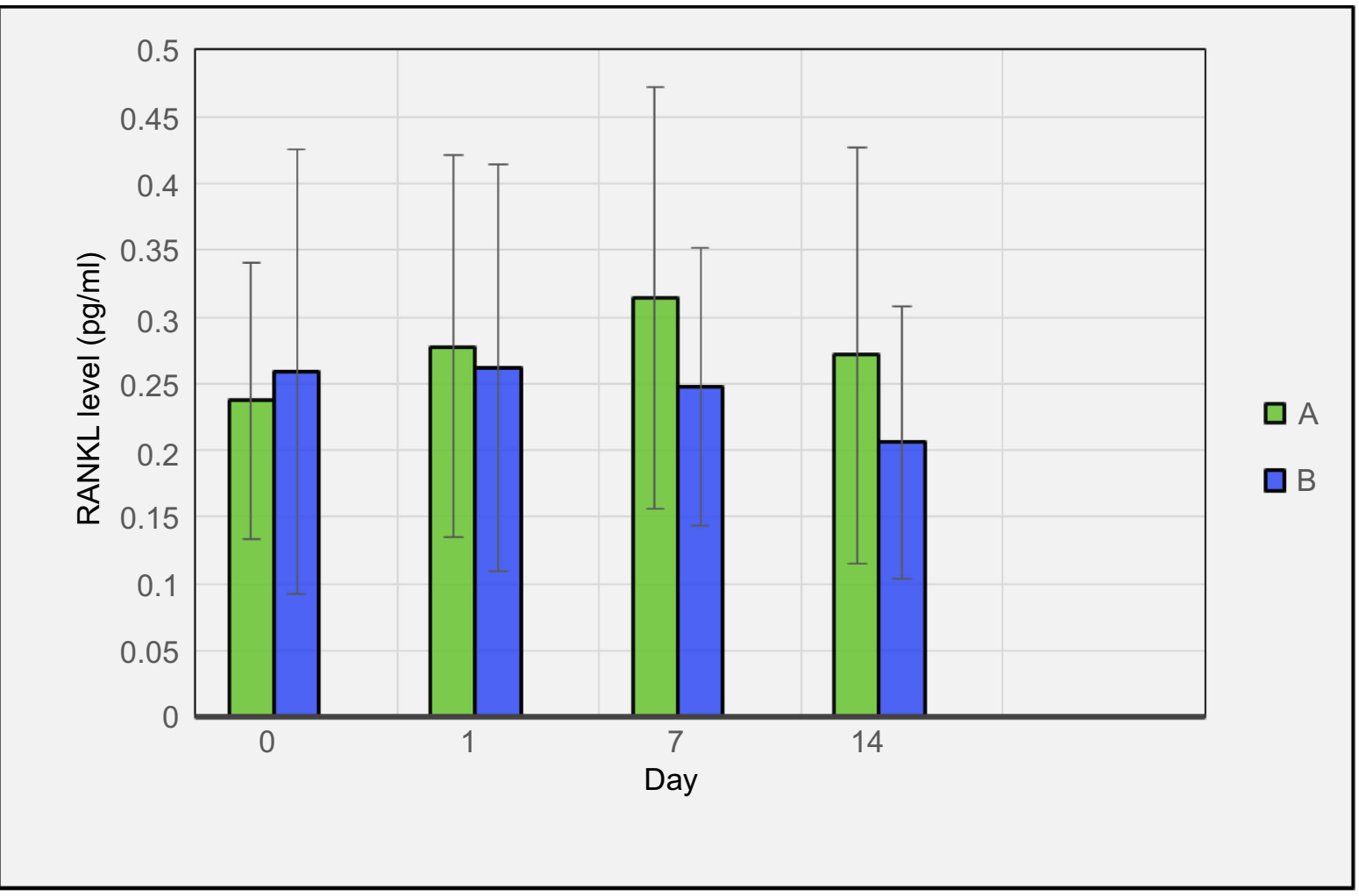

Figure 4 Comparison of RANKL level among 2 groups tested. A indicates control group, B indicates group receiving wheat seed.

Abbreviation: RANKL, receptor activator of nuclear factor- $\kappa \mathrm{B}$ ligand 


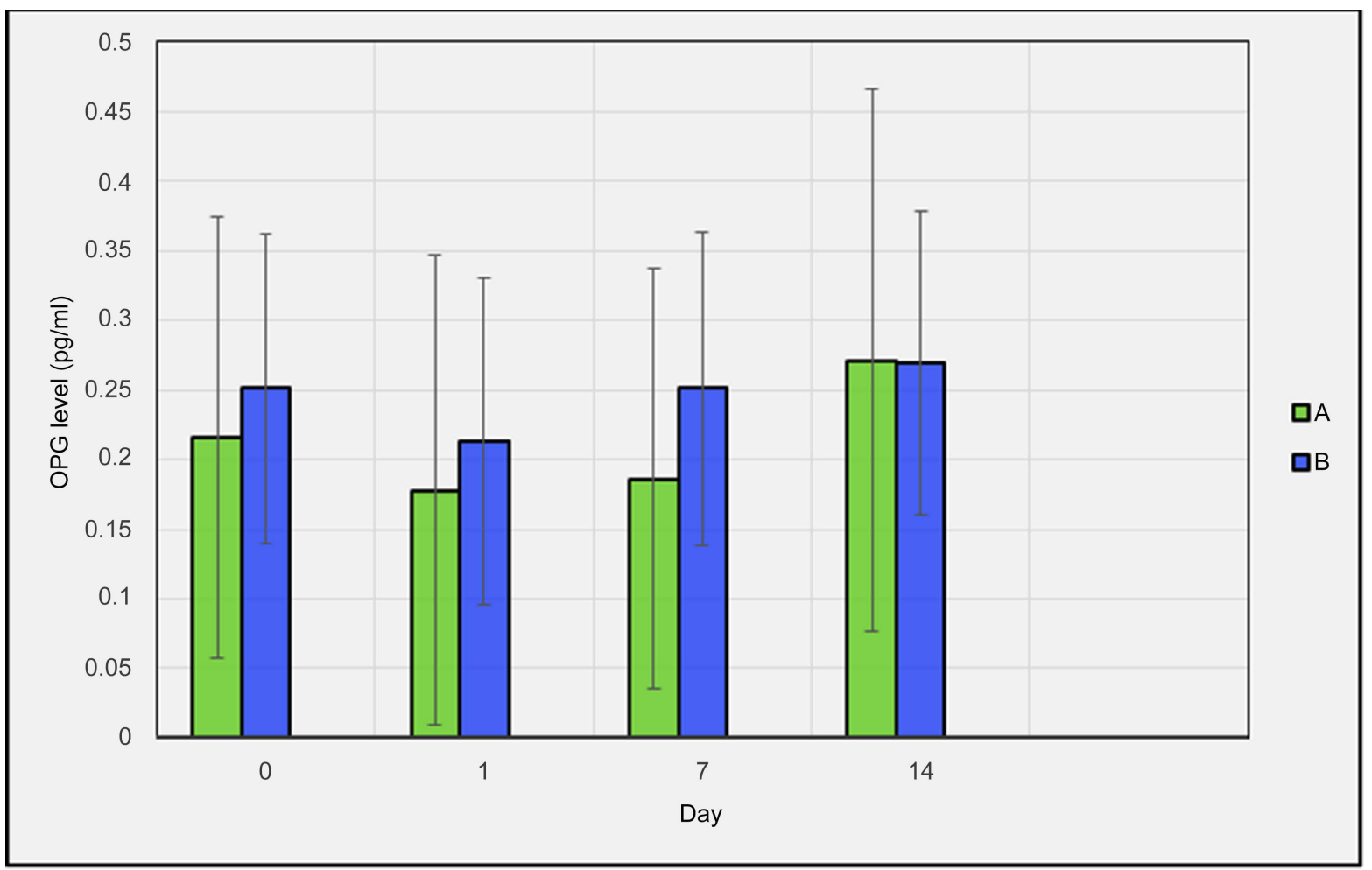

Figure 5 Comparison of OPG level among 2 groups tested. A indicates control group, B indicates group receiving wheat seed. Abbreviation: OPG, osteoprotegerin.

In this study, osteoprotegerin level elevated significantly and peaked on day 14 after orthodontic appliances debonding and wheat seed administration. A previous study found that estrogen can enhance osteoprotegerin expression in vitro and in vivo studies. ${ }^{13}$ Moreover, Jia et $\mathrm{al}^{14}$ stated that estrogen suppresses miR-145 expression, which has a role in regulating osteoblast differentiation. The reduction of miR-145 expression causes the release of OPG expression. In contrast, receptor activator of nuclear factor- $\mathrm{kB}$ ligand levels displayed a decreasing tendency following wheat seed treatment. RANKL leads osteoclast precursor cells (monocyte-lineage hematopoietic cell) to fuse with one another and become mature multinucleated osteoclast cells and indeed activating osteoclasts. $^{7}$ In response to reduced RANKL level, the decreased osteoclasts number induced. The decrease in the number of osteoclasts by giving wheat seeds can inhibit the tooth movement process during the orthodontic period of active tooth movement; therefore, the provision of wheat seeds, which can reduce the number of osteoclasts, is suggested in the passive period or retentive orthodontic treatment. ${ }^{8}$ The passive period is the treatment phase after the active period which aims to maintain the position of the teeth and supporting tissues in the new correct position and avoid teeth returning to their original positions. ${ }^{2}$ The administration of wheat seeds during the passive period of the orthodontic treatment is expected to maintain the tooth position after the active period of the orthodontic treatment.

\section{Conclusion}

The results of the present study demonstrated that wheat seeds administration might help to the passive phase of the OTM by blocking the rate of tooth movement, inhibiting the osteoclastogenesis (up-regulate OPG level and suppress RANKL level), and minimize the relapse after active orthodontic treatment. Further studies are necessary at clinical levels to confirm the efficacy and potency of wheat seeds in preventing relapse movement in orthodontic patients clinically.

\section{Limitation of study}

This is a pilot study with a small sample size and small scope of periodontal tissue examination. Larger sample size with a larger scope and bigger animal model mimicking the adapting relapse condition will provide more predictable results and validate the effect of wheat seeds for blocking relapse movement. 


\section{Acknowledgment}

This study project is entirely financed by grant Hibah Penelitian Dana Masyarakat, Faculty of Dentistry, Universitas Gadjah Mada, Republic of Indonesia, for the fiscal year 2018 following Contract No. 001461/KKEP/FKG-UGM/ $\mathrm{EC} / 2018$.

\section{Disclosure}

The authors report no conflicts of interest in this work.

\section{References}

1. Alhasyimi AA, Pudyani PS, Asmara W, Ana ID. Locally inhibition of orthodontic relapse by injection of carbonated hydroxyapatite advanced platelet-rich fibrin in a rabbit model. Key Eng Mater. 2017;758:255-263. doi:10.4028/www.scientific.net/KEM.758.255

2. Alhasyimi AA, Pudyani PS, Asmara W, Ana ID. Enhancement of postorthodontic tooth stability by carbonated hydroxyapatite-incorporated advanced platelet-rich fibrin in rabbits. Orthod Craniofac Res. 2018;21 (2):112-118. doi:10.1111/ocr.12224

3. Asefi S, Seifi M, Fard GH, Lotfi A. Innovative evaluation of local injective gel of curcumin on the orthodontic tooth movement in rats. Dent Res J. 2018;15:40-49. doi:10.4103/1735-3327.223618

4. Alhasyimi AA, Pudyani PS, Asmara W, Ana ID. Effect of carbonated hydroxyapatite incorporated advanced platelet-rich fibrin intramuscular injection on the alkaline phosphatase level during orthodontic relapse. AIP Conf Proc. 2018;1933(1):030006.

5. Avriliyanti F, Suparwitri S, Alhasyimi AA. Rinsing effect of $60 \%$ bay leaf (Syzygium polyanthum wight) aqueous decoction in inhibiting the accumulation of dental plaque during fixed orthodontic treatment. Dent J (Majalah Kedokteran Gigi). 2017;50(1):1-9. doi:10.20473/j. djmkg.v50.i1.p1-5
6. Hunsel F, Koppel S, Puijebbroek E. Post-menopausal vaginal hemorrhage related to the use of a hop-containing phytotherapeutic product. Drug Saf-Case Rep. 2015;2(14):5.

7. Kohli SS, Kohli VS. Role of RANKL-RANK/osteoprotegerin molecular complex in bone remodeling and its immunopathologic implications. Indian $J$ Endocrinol Metab. 2011;15(3):175-181. doi:10.4103/2230-8210.83401

8. Suparwitri S, Pudyani PS, Haryana SM, Agustina D. Effects of soy isoflavone genistein on orthodontic tooth movement in guinea pigs. Dent $J$ (Majalah Kedokteran Gigi). 2016;49(3):168-174. doi:10.20473/j.djmkg.v49.i3.p168-174

9. Kim H, Tabata A, Tomoyasu T, et al. Estrogen stimuli promote osteoblastic differentiation via the subtilisin-like proprotein convertase PACE4 in MC3T3-E1 cells. J Bone Miner Metab. 2015;33 (1):30-39. doi:10.1007/s00774-014-0567-9

10. Nimeri G, Kau CH, Corona R, Shelly J. The effect of photobiomodulation on root resorption during orthodontic treatment. Clin Cosmet Investig Dent. 2014;6:1-8.

11. Patisaul HB, Jefferson W. The pros and cons of phytoestrogens. Front Neuroendocrinol. 2010;31(4):400-419. doi:10.1016/j.yfrne.2010.01.003

12. Alhasyimi AA, Rosyida NF. Cocoa administration may accelerate orthodontic tooth movement by inducing osteoclastogenesis in rats. Iran J Basic Med Sci. 2019;22(2):206-210. doi:10.22038/ ijbms.2018.29024.7021

13. Manokawinchoke J, Ritprajak P, Osathanon T, Pavasant P. Estradiol induces osteoprotegerin expression by human dental pulp cells. Odontology. 2016;104:10-18. doi:10.1007/s10266-014-0178-x

14. Jia J, Zhou H, Zeng X, Feng S. Estrogen stimulates osteoprotegerin expression via the suppression of miR-145 expression in MG-63 cells. Mol Med Rep. 2017;15(4):1539-1546. doi:10.3892/ mmr.2017.6168
Clinical, Cosmetic and Investigational Dentistry

\section{Publish your work in this journal}

Clinical, Cosmetic and Investigational Dentistry is an international, peer-reviewed, open access, online journal focusing on the latest clinical and experimental research in dentistry with specific emphasis on cosmetic interventions. Innovative developments in dental materials, techniques and devices that improve outcomes and patient

\section{Dovepress}

satisfaction and preference will be highlighted. The manuscript management system is completely online and includes a very quick and fair peer-review system, which is all easy to use. Visit http://www.dovepress.com/testimonials.php to read real quotes from published authors. 